\title{
Differences in Renal Transplantation in India and First World Countries
}

\author{
M. N. Mishra ${ }^{1}$, V.K. Saxena ${ }^{2}$ and A.S. Narula ${ }^{3}$ \\ ${ }^{1}$ Department of Pathology, ${ }^{2}$ Department of Urology, ${ }^{3}$ Department of Nephrology \\ INHS Asvini, Colaba, Mumbai 400 005, Maharashtra, India
}

KEYWORDS Kidney transplantation, acute rejection; donor profile

\begin{abstract}
A retrospective study was carried out at INHS Asvini to analyse certain aspects of kidney transplantation and compare the data generated with available data from developed countries in literature. The etiology of end stage renal disease, age and sex distribution of recipients, donor profile, HLA typing results, episodes of acute rejection, causes of post transplant morbidity and mortality were studied and compared with some of the other contemporary studies. Males outnumbered females, as recipients while the number of female donors was more. All 6 alleles were identified in only sixty- four samples by microlymphocytoxicity-based methods while by DNA based HLA typing all six alleles were identified in nearly $80 \%$ patients with class II in over $90 \%$ cases. In this study perioperative causes and infections led to loss of graft in five patients each.
\end{abstract}

\section{INTRODUCTION}

Kidney transplantation although not a panacea for patients with end stage renal diseases (ESRD), is the only long-term solution. Most kidney transplants are performed in India (Panigrahi et al. 2002) from a living donor- unlike the west where cadaver transplants are much more common (Dyer et al. 1989; Foss et al. 1998). This study includes data pertaining to 175 kidney recipients at INHS Asvini . The study included eight retransplants and 167 first time recipients. Commerce in kidney transplantation has been reported previously from India (Ivanovski et al. 1997; Server et al. 1995). We studied the donorrecipient profile, underlying primary disease, HLA typing results, incidence of acute rejection (AR) and causes of graft loss. The results are compared with those available in literature. Unlike the west where there is no overwhelming preponderance of any particular sex among donors and recipients we found that females outnumbered males as donors but comprised only $15 \%$ of recipients. The commonest diagnosis of ESRD was chronic glomerulonephritis -essentially a diagnosis by exclusion and based on the radiological findings, with a specific cause detected in only $15 \%$ of cases. All six alleles of A, B and DR antigens could be detected only in $64 / 230$ samples by serology. Only 15 recipients experienced biopsy proven AR. The clinically suspected cases of AR are not included in the data. The 3-month graft survival rate was above $90 \%$. Infections resulted in loss of six grafts due to death of patients with a functional graft. Graft loss due to infection is common in developing countries (Rizvi and Nagvi 2001; Mishra et al. 1999) while in developed countries immunological causes probably account for graft loss as evidenced by improved graft survival with increased HLA match (Panigrahi et al. 2002; McKenna et al. 1998; Tajik et al. 2001).

\section{MATERIALS AND METHODS}

\section{HLA Typing}

Blood samples were collected in 10-15 units of phenol free liquid sodium heparin $10^{5}$ international units/ $\mathrm{ml}$ from each patient and donor for serological typing. Lymphocytes were isolated by density gradient centrifugation using Lymphoprep (Nycomed \& Co, Oslo, Norway). B and $\mathrm{T}$ cell separation was done using nylon wool column (Robbins.Scientfic, California, USA) based on the principle that $\mathrm{T}$ lymphocytes do not adhere to nylon wool which can therefore be eluted out by washing the nylon wool columns with tissue culture fluids. B-lymphocytes that remain adherent to nylon wool can be harvested by gentle squeezing of the columns after washing with RPMI/FCS (Gibco, Flow Laboratories) at $4^{0} \mathrm{C}$. HLA typing was done by NIH two-stage microlymphocytotoxicity assay (Terasaki and 
McClleland 1964) using commercial antisera from (BAG, Germany and Pelfreeze USA). Microlymphocytoxicity based tissue -typing was done for 230 donors and recipients while 120 samples were typed by molecular methods.

DNA had been extracted from whole blood collected in EDTA by salting out method for seventy samples and using column based extraction using Qiagen (Germany) kits for remaining 50 samples. DNA based molecular typing of Class II HLA antigens for 45 patients and their donors was done by Sequence Specific Primers (SSP) using (Genovision Olerup, USA) SSP intermediate resolution kit and for 25 patients and their donors using Biotest Kits (Biotest AG, Landsteinerstrasse Dreieich, Germany). The procedure mentioned in the product insert was followed. Briefly the steps were initial denaturing at $94^{\circ} \mathrm{C}$ for 2 minutes followed by deanturation at $94^{\circ} \mathrm{C}$ for 10 seconds; annealing and extension at $65^{\circ} \mathrm{C}$ for 60 seconds ( 10 cycles) followed finally by deanturation at $94^{\circ} \mathrm{C}$ for 10 seconds; annealing at $61^{\circ} \mathrm{C}$ for 50 seconds and extension at $72^{\circ} \mathrm{C}$ for 30 seconds ( 20 cycles). The PCR products were identified using agarose gel electrophoresis followed by detection of DNA bands in ultra violet light using a gel documentation system (UVP, England).

\section{Donor Specific Cross-match}

For all donors-recipient pairs, donor specific crossmatch was done twice, once at the time of HLA typing and repeated 48 hrs before transplantation. A cross-match result was taken as positive if the percentage of dead cells exceeded $20 \%$ of the negative controls. Donor peripheral blood lymphocytes were isolated using nylon wool and microlymphocytotoxicity based crossmatch was done. B cell crossmatch was done in patients who had crossmatch results exceeding $15 \%$ and those requiring second graft. Three patients with initial positive B cell crossmatch who later became negative underwent renal transplantation. Panel reactive antibody testing was not included in the usual immunological workup of our patients and the same was done only for three patients with an initial B cell crossmatch positive result.

\section{Immunosuppression}

The immunosuppression protocol at our center is: Day -2 to 0 Cyclosporine (Cs) $8 \mathrm{mg} / \mathrm{kg}$ orally, Azathioprine $1 \mathrm{mg} / \mathrm{kg}$, Injection Methyl Prednisolone $500 \mathrm{mg}$ Intravenously (IV) on day -1 and after relieving clamps. Day 1 onwards Cs $8 \mathrm{mg} / \mathrm{kg}$, tapered by $1 \mathrm{mg} / \mathrm{kg}$ per month to a maintenance dose of $3 \mathrm{mg} / \mathrm{kg}$; Azathioprine 1mg/ $\mathrm{kg}$ and Prednisolone $30 \mathrm{mg}$ once a day, tapered by $5 \mathrm{mg}$ every month to a maintenance dose of $10 \mathrm{mg} /$ day. All the three drugs are administered life long. AR episodes were treated with Methyl Prednisolone initially in a dose of $0.5 \mathrm{~g}$ intravenously/day for 3 days. Refractory cases of biopsy confirmed AR was treated with either OKT-3 or Anti-thymocyteglobulin.

\section{Needle Biopsy and Surgical Pathology}

After suspicion of clinical AR, biopsy was done under ultrasound guidance using Monopty Semiautomatic Biopsy Gun with cutting edge (Bard Inc) and histological diagnosis was made according to the Banff working classification (Racusen et al. 1999). Twenty- six needle biopsies, eight graft nephrectomies and two postmortem specimen of donor kidneys were subjected to histopathological examination. All tissue was collected and dispatched to the laboratory in 10 $\%$ formal saline followed by routine preparation for paraffin embedded sections. Twenty- five sections of 3-5 $\mu$ thick sections were cut and stained with hematoxylin and eosin, periodic acid Schiff reagent (PAS) and periodic acid silver methamine stains (PAM). A graft failure was defined as a loss of kidney function but also included recipient deaths with functional graft.

\section{RESULTS}

The donor-recipient profiles of 175 transplants are shown in Figure 1. One hundred donors were first-degree relatives - live related donors (LRD) of the recipients. This included mother $(n=37)$, father $(n=17)$, brother $(n=29)$, sister $(n=14)$, and son $(n=1)$. Seventy- five of the recipients were non - first-degree relatives of the recipients (LURD) and included wife ( $\mathrm{n}=$ $43)$, cousin $(n=11)$, uncle $(n=7)$, in-laws $(n=5)$, husband $(n=4)$, nephew $(n=3)$ and aunt $(n=2)$. There was a female preponderance among donors $(60 \%)$ and male preponderance among recipients (83\%). Since 1997 there has been an increase in transplants from biologically unrelated donors and the wife of the recipient has emerged as very common donor. (Fig. 1) When spousal transplants were considered as a subset, out of 
47 transplants only four $(8 \%)$ were from husband to wife.

The commonest HLA alleles were A9, A24, A1; B5, B40; DR1, DR2. All six HLA antigens were identified only in $28 \%$ of individuals, with maximum number of blanks in DR typing on serology. The quality and quantity of DNA extracted from whole blood using Qiagen kits was satisfactory for SSP. The results of SSP typing for DR antigens were much superior and both alleles could be detected in $90 \%$ of persons $(\mathrm{n}=120)$ and all six alleles in $85 \%$ samples. There were only 6 patients with zero mismatch, 69 patients were mismatched for 5 or 6 alleles and 100 patients were mismatched for 3 or 4 alleles (Table 1). All donor - recipients were crossmatch negative.

Kidney biopsy was done in 26 patients who had increased serum creatinine to establish the cause of altered renal function. Of the 15 cases of AR, 10 were partially reversed by anti-rejection treatment. Two cases of AR were due to vascular rejection, the remaining were due to cellular rejection. Needle biopsy was done for 26 cases with poor renal function, the diagnosis of which is given in table 2 . The histopathological findings included acute cellular rejection $(n=13)$, vascular rejection $(n=2)$ : infarction $(n=3)$, inadequate $(n$ $=1)$, normal $(n=2)$, borderline changes of AR and chronic rejection $(\mathrm{n}=2$,each). One patient showed striped fibrosis - evidence of

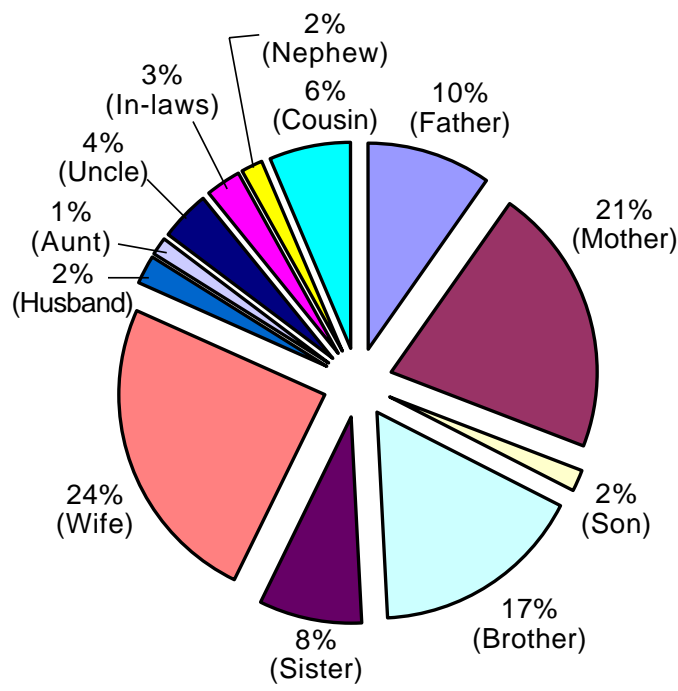

Fig. 1. Donor profile cyclosporine toxicity. The postmortem specimen of kidney was normal on histological examination. The nephrectomy specimen showed infarction $(n=4)$ and evidence of chronic graft nephropathy $(n=4)$ thus confirming the initial diagnosis made on needle biopsy (Table 3 ). The patients with a normal needle biopsy succumbed to infection and the allograft was found to be normal on histopathological examination of post mortem specimen.

The follow-up period of patients varied from 6 months to 5 years during this period a total of 20 grafts were lost. The complete data is not available as many of our patients are transferred back to a hospital close to their native place after three months after transplantation. In all twenty grafts were lost. Four patients had graft infarction; two grafts failed due to severe vascular rejection, one had graft rupture and three had lost their grafts due to perioperative mortality. Three more patients had chronic rejection of

Table 1: Number of HLA allele mismatch between recipient - donor pairs

\begin{tabular}{lcc}
\hline Mismatch & LRD $=100$ & LURD $=75$ \\
\hline$<3$ & 6 & 0 \\
3 & 58 & 11 \\
4 & 11 & 20 \\
5 & 15 & 14 \\
6 & 10 & 30 \\
\hline
\end{tabular}

Legend: LRD - Live related donation, LURD - Live unrelated donation

Table 2: Biopsy diagnosis of 26 suspected cases of $\mathbf{A R}$

Acute Cellular Rejection 13

Acute Vascular Rejection 2

Chronic Rejection

Striped fibrosis (Cyclosporin Toxicity)

Borderline changes

Inadequate

Infarctions

Normal

2
2
1
2
1
3
2

Table 3: Causes of graft loss $n=23$

\begin{tabular}{lc}
\hline Etiology & Number \\
\hline Bacterial Septicemia & 4 \\
Mycosis & 1 \\
Ludwig's angina & 1 \\
P-falciparum & 1 \\
Graft Rupture & 1 \\
Chronic Rejection & 8 \\
Infarction & 3 \\
Immunological causes & 4 \\
\hline
\end{tabular}


insidious onset and returned to maintenance hemodialysis. Another ten patients were having slight renal function compromise as their serum creatinine was persistently $>2.5 \mathrm{mg} / \mathrm{dl}$.

\section{DISCUSSION}

Most kidney transplants in India take place from a living donor to the recipient. Cadaver donation has not become very popular in India. In India the Organ Donation Bill gazette notification by the Ministry of Health and Family Welfare, Government of India on 04 Feb 1995 was a landmark event in that it allowed removal of organs from deceased and live unrelated donors. Cadaver organ donation is yet to become popular in India and often articles appear in lay press regarding unscrupulous commerce in kidneys. Some patients and relatives confide being approached by middlemen for sale of kidneys. At All India Institute of Medical Sciences it commenced in 1993, but only 25 cadaver organ donations occurred (Panigrahi et al. 2002) in the center till 1999. In Jan 2003 for the first time two centers in different cities (Bangalore and Mumbai) shared organs from the same cadaver. Endeavor should be directed to popularize cadaver donation, as there will be a much bigger reservoir of kidneys available and this alone shall curb unscrupulous trade. Cadaver organ donations are gradually becoming popular in South India. Only 272 cadaver renal transplants had been performed in India till 1999 (Shah 2001) and organizing cadaver donors in India is always a problem (Shankarkumar 2001). Today with advances in immunosuppression the results of live related and cadaver donations are comparable in terms of graft survival and in fact many patients who receive kidneys from biologically unrelated donors do better than those who receive kidneys from cadaver with a higher degree of match (Foss et al. 1998; Terasaki et al. 1995; Alfani et al. 1997).

Male recipients $(87 \%)$ outnumbered female recipients, whereas female donors outnumbered males in the ratio 2: 1 in this study, which is similar to that of Panigrahi et al. (2002) where $86 \%$ of recipients was males. Chronic renal failure is not known to have any predilection for males. In Indian society male is still the main bread earner and probably more exposed to diseases. However economic reasons alone can't explain the much lower incidence of female patients with ESRD seeking medical aid, as the treatment is free at our center. Of the 26 female kidney recipients in the study, four received a kidney from their husbands and eight from their mothers. In most western studies female recipients is higher and they comprised 25-40\% of all recipients (Foss et al. 1998; McKenna et al. 1998).

In the study 26 episodes of suspected AR occurred $(15 \%)$ of which there were 3 cases of graft infarction and one of cyclosporine toxicity. One of the biopsies was inadequate whereas two had borderline changes. AR was confirmed in 15 cases on biopsy. Of the 17 cases, one was vascular rejection; one of humoral rejection and 15 were cases of cellular rejection. This figure is much lower than that in western literature where figures range from 40- 60\% (Midtvedt et al. 1998; Kanetsuna et al. 2003) and this cannot be explained only by the fact that most kidney donations in India are from a living donor. The probability of subcliniical cases being missed cannot be excluded. Histopathological examination of the allograft is still the definitive diagnostic tool for diagnosis of AR (Shmori et al. 2000). Only $1 / 26$ sample of needle biopsy was inadequate. Some authors recommend protocol biopsy for early detection of subclinical cases (Kanetsuna et al. 2003). The answer can come by doing protocol biopsy on a large population of allograft recipients.

HLA typing was done for 230 samples (165 recipients and their donors) by serology and only in sixty-four cases were all six alleles identified; both the DR antigens could be identified only in $60 \%$ of cases. Our findings were similar to that of Shankarkumar et al. (1999) have also reported high frequency of A9, A24, B5 and B7 in their study on HLA antigen distribution in Marathi population. The results of DR typing by generic molecular methods were far superior and both alleles were identified in $90 \%$ cases. Thus there is a requirement to switch over to generic molecular typing by SSP or by Reverse hybridization.

The causes of postoperative morbidity were infections from which the patients recovered (Varicella zoster -3, bacterial pneumonia and disseminated candida infection - 1 each) and graft infarction $(\mathbf{n}=3)$ and graft rupture $(\mathbf{n}=1)$. Severe bacterial septicemia led to seven recipient deaths; four succumbed to bacterial septicemia, one each died of $P$-falciparum malaria, Ludwig's angina and disseminated mycosis. Four patients lost their grafts due to immunological causes - 
two due to accelerated rejection and two went into maintenance hemodialysis (MHD) shortly after partial reversal of acute rejection episode. Three patients developed graft infarction shortly after surgery and one graft ruptured. In these patients too immunological causes such as antiphospholipid antibodies or endothelial cell antibodies cannot be excluded, as they were not looked for. Although the patients often die with a functional graft, in actual terms this is a graft loss and must be categorized as such. The incidence of graft loss in the study by Panigrahi et al. (1) due to infection was 14/42 LURD and 15/ 25 in patients who had received a cadaver transplant during five year follow up.

The incidence of graft loss in India due to infections is higher than the west where the main cause for graft loss is immunological. The possible cause for such high incidence of infection could be due to use of steroid based immunosuppression regimen used in India. The long-term graft survival rate can be improved by modifying the immunosuppression therapy and closer immunological monitoring.

\section{CONCLUSION}

In conclusion we advocate early biopsy in patients with renal disease to know the etiology, a more comprehensive immunological pretransplant workup including testing for antiphospholipid antibodies, B cell crossmatch, DNA based typing and closer post transplant monitoring including protocol biopsies along with no or low steroid immunosuppressive regimen for better graft outcome.

\section{ACKNOWLEDGEMENT}

The authors are grateful Dr Mrs. SV Trikkanad and Dr Mrs. SR Kannkonkar for carrying out crossmatch of all our patients and tissue typing for 300 samples on payment of the stipulated cost.

\section{REFERENCES}

Alfani D, Pretagostini R, Rossi M, Poli L et al. 1997. Analysis of 160 consecutive living unrelated kidney transplants: 1983-1997. Transplant Proc, 29: 339901.

Dyer AP, Martin S, Mallick NP, Gokal R, Johnson RWG 1989. Kidney transplantation in the northwest region of England - An experience of 1132 trans- plants in 21 years. In: PI Terasaki (Ed.): Clinical Transplants 1989. Los Angeles: UCLA Tissue Typing Laboratory. Pp 205.

Foss A, Leivstead T, Brekke IB et al. 1998. Unrelated living donors in 141 kidney transplantations. Transplantation, $66(1)$ : 49-52

Ivanovski N, Stojkovski L et al. 1997. Renal transplantation from paid and unrelated donors in

India - it is not only unethical, it is also medically unsafe. Nephrol Dial Transplant, 12: 13.

Kanetsuna Y, Yamaguchi Y, Toma H, Tanabe K 2003. Histological evaluation of renal allograft protocol biopsies in early period and one year after transplantation. Clin Transpl, 17 (S10): 25-9.

McKenna RM, Lee KR, Gough JC et al. 1998. Matching for private or public epitopes reduces acute rejection episodes and improves two - year renal allograft function. Transplantation, 43 (1): 38 - 43.

Midtvedt K, Hartmann A, Snud S 1998. Can a diagnosis of renal allograft rejection be based on histology alone? Clin Transpl, 12: 300-02.

Mishra MN, Panigrahi A, Kanga U et al. 1999. Unrelated renal transplantation: A study of 71 consecutive cases. Proceedings ASEATTA, Australia.

Panigrahi A, Agarwal SK, Kanga U et al. 2002. Influence of HLA compatibility on renal graft survival using live unrelated and cadaver donors in India. Indian $J$ Med Res, 115: 158-64.

Racusen LC, Solez K, Colvin RB et al. 1999. The Banff 97 working classification of renal allograft pathology. Kidney Int, 55 (2): 713.

Rizvi A, Naqvi A 2001. Current issues and future problems in transplantation in East Asia. Transplant Proc, 33: $2623-5$.

Sever MS et al. 1995. Living unrelated kidney transplantation in India; high risk of complications other than the well known ethical problems. Kidney Int, 48 (5): 1679.

Shah VB. 2001. Editorial in Narmada Kidney Foundation Newsletter (15-16): 1.

Shankarkumar U 2001. HLA matching and cadaver renal transplantation in India. $\mathrm{Br} J$ Biomed $S c i, \mathbf{5 8 ( 4 )}$ : 255.

Shankarkumar U, Pednaker SV, Gupte S, Ghosh K, Mohanty D 1999. HLA antigen distribution in Marathi speaking Hindu population from Mumbai, Maharastra. J Hum Ecol, 10 (5-6): 367-72.

Shmori K, Kuratatae I, Sakatani T et al. 2000. Histopathology of a human allografted kidney with clinically sufficient function. Clin Transpl, 14 (S3): 25-9.

Tajik N, Singal DP, Pourmad G et al. 2001. Prospective study of microchimerism in renal allograft recipients:between HLA - DR matching, microchimerism and acute rejection. Clin Transplantation, 15: $192-8$

Terasaki PI, Mc Clelland JD 1964. Microdroplet assay of human serum cytotoxins. Nature, 204: 9981000 .

Terasaki PI, Cecka M, Gjertson DW, Takemoto S 1995. High survival rates of kidney from spousal and unrelated donors. N. Engl J Med, 333: 333-6. 